\title{
Neurocriptococose em paciente imunocompetente: um relato de caso
}

\author{
Neurocriptocosis in an immunocompetent patient: a case report
}

Neurocriptocosis en un paciente inmunocompetente: un reporte de caso

Sylvia Rannyelle Teixeira Lima ${ }^{1 *}$, Larissa Marley Ribeiro de Sousa ${ }^{2}$, João Kennedy Teixeira Lima ${ }^{3}$, Antônio Leonel de Lima Júnior ${ }^{4}$.

\section{RESUMO}

Objetivo: Relatar caso raro de neurocriptococose em paciente imunocompetente. Detalhamento de caso: A infecção do sistema nervoso central pelo fungo Cryptococcus neoformans, também chamada neurocriptococose, é uma desordem rara mais comum em pessoas que possuem algum grau de comprometimento imunológico, seja primário ou secundário, atuando como infecção de caráter oportunista. Descrevemos um caso de paciente imunocompetente com achados laboratoriais compatíveis com neurocriptococose que se apresentava apenas com cefaleia de características migranosas, nódulo pulmonar isolado sem queixas clínicas e exames de imagem de sistema nervoso central inespecíficos. Considerações finais: Em pacientes imunocompetentes, infecções fúngicas são raras e podem cursar com sintomatologia não evidente, dificultando o diagnostico etiológico. É necessária história clínica minuciosa associada a exames complementares bem empregados com o intuito de chegar ao diagnostico etiológico de forma mais segura possível para assegurar a terapêutica correta. Ademais, se faz necessário emprego de técnicas mais rápidas e acessíveis ao diagnostico, como também para triagem, visto que parte desses pacientes são oligossintomaticos e se beneficiariam de tratamento precoce.

Palavras-chave: Meningite criptococócica, Cryptococcus neoformans, Meningoencefalite.

\begin{abstract}
Objective: To report a rare case of neurocriptococcosis in a immunocompetent patient. Case report: The infection of the central nervous system by the fungus Cryptococcus neoformans, also called neurocriptococcosis, is a rare disorder more common in people who have some degree of immune impairment, whether primary or secondary, acting as an opportunistic infection. We describe a case of an immunocompetent patient with laboratory findings compatible with neurocriptococcosis who presented only with migraine headache, isolated pulmonary nodule without clinical complaints and nonspecific central nervous system imaging. Final considerations: In immunocompetent patients, fungal infections are rare and may present with non-evident symptoms, making the etiological diagnosis difficult. Thorough medical history associated with complementary tests well used is necessary in order to arrive at the etiological diagnosis as safely as possible to ensure the correct therapy. In addition, it is necessary to employ the fastest techniques and obtain diagnosis, as well as for screening, since these patients are oligosymptomatic and benefit from early treatment.
\end{abstract}

Keywords: Meningitis cryptococcal, Cryptococcus neoformans, Meningoencephalitis.

\footnotetext{
1 Universidade Federal do Cariri (UFCA), Juazeiro do Norte - CE.

*E-mail: sylviarannyelle@gmail.com

2 Faculdade de Medicina de Juazeiro do Norte (FMJ), Juazeiro do Norte-CE.

${ }^{3}$ Faculdade de Medicina do ABC (FMABC), São Paulo - SP.

${ }^{4}$ Universidade Estadual do Ceará (UECE), Fortaleza - CE.
} 


\section{RESUMEN}

Objetivo: Reportar un caso raro de neurocriptococosis en un paciente inmunocompetente. Detalles del caso: La infección del sistema nervioso central por el hongo Cryptococcus neoformans, también llamado neurocriptococosis, es un trastorno poco común más común en personas que tienen algún grado de deterioro inmunológico, ya sea primario o secundario, que actúa como una infección oportunista. Describimos el caso de un paciente inmunocompetente con hallazgos de laboratorio compatibles con neurocriptococosis que se presentó únicamente con cefalea migrañosa, nódulo pulmonar aislado sin quejas clínicas e imagen inespecífica del sistema nervioso central. Consideraciones finales: En pacientes inmunocompetentes, las infecciones fúngicas son raras y pueden presentarse con síntomas no evidentes, lo que dificulta el diagnóstico etiológico. Es necesario un historial médico completo asociado a pruebas complementarias bien utilizadas para llegar al diagnóstico etiológico de la forma más segura posible y asegurar la correcta terapia. Además, es necesario emplear las técnicas más rápidas y obtener el diagnóstico, así como para el cribado, ya que estos pacientes son oligosintomáticos y se benefician de un tratamiento precoz.

Palabras clave: Meningitis criptocócica, Cryptococcus neoformans, Meningoencefalitis.

\section{INTRODUÇÃO}

Definida como infecção do sistema nervoso central causada pelo fungo encapsulado Cryptococcus neoformans, a neurocriptococose é uma entidade clínica comum em pacientes com Síndrome da Imunodeficiência Humana Adquirida (SIDA) ou pacientes com algum grau de imunossupressão. Em pacientes imunocompetentes é considerada rara e pode gerar equívoco na etiologia das afecções meningoencefálicas (JHA A, et al., 2019; NIKNAM N, et al., 2014).

Nesse contexto, para o diagnóstico da meningoencefalite pelo Criptococo, além do quadro clínico de meningoencefalite associada a alteração pulmonar, na maioria dos casos sintomáticos, são necessários exames complementares. Dado o risco aumentado de biopsia cerebral, a análise do líquido cefalorraquidiano é de fato um bom preditor, quando solicitado em momento oportuno com cultura, citologia, teste do látex para Cryptococcus neoformans, cultura em ágar Sabouraud e a confirmação pode ser feita com o uso de "tinta da China". Outra opção viável seria o lavado broncoalveolar com histopatologia do material que, obtendo-se amostras de lesões de vias aéreas, a biopsia endobronqueal está indicada (GOLDMAN JD, et al., 2010; MACHADO LR, et al., 2013)

Nos exames de imagem, a tomografia de tórax pode auxiliar no diagnóstico diferencial das afecções pulmonares relacionadas a fungos. $\mathrm{O}$ uso da ressonância magnética fica restrito à neuroimagem em que apresenta cistos heterogêneos com edema vasogênico ao redor, levando ao diagnostico diferencial de uma infecção fúngica (GOLDMAN JD, et al., 2010).

Em relação ao tratamento, apesar de divergências na literatura em relação ao tempo, é bem estabelecido o uso da anfotericina B lipossomal e fluconazol, ambos feitos em ambiente hospitalar a priori com posterior manutenção apenas com o fluconazol e analise de líquor cefalorraquidiano de controle (DIENSTMANN G, et al., 2019; GOLDMAN JD, et al., 2010; NIKNAM N, et al., 2014).

Este trabalho tem como objetivo apresentar um caso raro de neurocriptococose em paciente imunocompetente ocorrido em hospital brasileiro, com diagnóstico e tratamentos feitos com equipe multidisciplinar. Dessa forma, avaliar os diagnósticos diferenciais de meningoencefalites trará benefícios para possíveis futuros diagnósticos etiológicos e, dessa forma, contribuir para auxiliar no manejo destes pacientes.

\section{DETALHAMENTO DE CASO}

Trata-se de um caso de paciente do sexo feminino, parda, 55 anos, natural e procedente de interior do Ceará, que se internou em hospital de referência da região com história de cefaleia em região frontal e retro ocular de caráter pulsátil, associada a fotofobia, fonofobia e náuseas há 18 anos. Descreve que há 4 meses houve piora do quadro com aumento da intensidade e frequência de episódios, com prejuízo do sono, apetite e humor. 
Procurou neurologista ambulatorialmente que solicitou exames de imagem e laboratoriais. Dentre estes, encontrava-se uma análise de liquor cefalorraquidiano com citologia de 5 hemácias $p / \mathrm{mm} 3,15$ leucócitos $\mathrm{p} / \mathrm{mm} 3$, com diferencial: linfomononucleares $16 \%$ polimorfonucleares $84 \%$ Monócitos $0 \%-100 \%$. A bioquímica apresentava glicose $13 \mathrm{mg} / \mathrm{dl} \mathrm{Cl} 112 \mathrm{mEq} / \mathrm{l}$ proteinas $166 \mathrm{mg} / \mathrm{dl}$. Reação Pandy +/1 Reação de Nonne $+/ 2$ Ziehl-Nilelsen, pesquisa de cryptococcus VDRL e Gram negativos. Cultura negativa. Pesquisa de células neoplásicas negativa.

Ademais, possuía ressonância magnética de crânio externa evidenciando discreta tortuosidade e proeminência dos espaços liquoricos ao redor dos nervos óticos, insinuação inferior das tonsilas cerebelares $0,3 \mathrm{~cm}$ abaixo pelo forame magno e diminutos focos de hiperssinal em T2 e FLAIR esparsos pela substância branca dos hemisférios cerebrais, inespecíficos, podendo representar focos de gliose / migrânea/ rarefação da mielina. Radiografia de tórax evidenciando opacidade mal definida em terço médio de hemitórax direito.

À admissão hospitalar, referia a cefaleia de mesmas características e perda de peso de aproximadamente 5 quilos em 4 meses. Negava sintomas de febre, dispneia, tosse, hemoptise, hematêmese, diarreia, disúria, mialgia e artralgia. História pregressa de hipertensão e enxaqueca. Referia contato com pássaros em seu domicílio.

Ao exame físico encontrava-se com bom estado geral, anictérica, acianótica, afebril, corada. Ausculta respiratória e cardíaca sem alterações, abdome sem achados. Ao exame neurológico, encontrava-se lúcida, consciente e orientada, sem achados de rigidez de nuca (Testes de Brudzinski e Lasegue negativos), reflexos tendinosos presentes e simétricos, pupilas isocóricas e fotorreagentes e Escala de Coma de Glasgow 15.

Diante do exposto, fora então solicitada nova tomografia de tórax e iniciada antibioticoterapia com ceftriaxona $2 \mathrm{~g}$ de $12 / 12$ horas. A tomografia apresentava opacidade nodular com atenuação de partes moles de limites definidos, margens levemente lobuladas, com leve realce difuso, localizada em região subpleural do segmento superior do lobo inferior direito, associado a bronquiolectasias, medindo $2,8 \times 2,4 \times 1,6 \mathrm{~cm}$ (LL $x$ AP x CC).

Após parecer da infectologia, achou-se pertinente nova coleta de LCR com cultura para fungos, bactérias, VDRL e ADA, além de ressonância magnética de crânio para melhor avaliação dos achados do resultado do exame externo. Ademais, a pesquisa de imunodeficiência (síndrome da imunodeficiência humana, neoplasias, colagenoses) foi indicada.

A ressonância magnética apresentou hiperssinal difuso na sequência FLAIR no espaço subaracnóideo (LCR) de etiologia inespecífica que, levando em consideração a hipótese de meningoencefalite, poderá estar relacionada a aumento do teor proteico no líquor em processos inflamatórios, além de focos esparsos de alteração de sinal em sustância branca subcortical e profunda, de aspecto inespecífico, podendo corresponder a gliose por microangiopatia (Fazekas 1). Exames laboratoriais, apresentando fator antinuclear (FAN) não reagente, sumário de urina tipo 1 (EAS) com 8 leucócitos por campo e eletroforese de proteínas sem alterações, e sorologia para HIV 1 e 2 negativas.

O diagnóstico etiológico surgiu após resultado do LCR que apresentou -se límpido e incolor com citologia de hemácias 2 cél $/ \mathrm{mm} 3$ leucócitos 585 cél $/ \mathrm{mm} 3$ neutrófilos $78 \%$ Segmentados $78 \%$ Bastões $0 \%$ Eosinófilos $0 \%$ basófilos $0 \%$ linfócitos típicos $22 \%$ sem células atípicas. Valores de glicose $20 \mathrm{mg} / \mathrm{dl}$ cloreto $112 \mathrm{mEq} / \mathrm{l}$ proteínas totais $229 \mathrm{mg} / \mathrm{dl}$. A cultura automatizada mostrou isolado 1 e para fungos: Cryptococcus sp (sensível à anfotericina B). A pesquisa para detecção de fungos revelou: presença de estruturas leveduriformes compatíveis com o gênero Cryptococcus sp. BAAR e VDRL negativos.

Feito o diagnóstico de neurocriptococose em paciente sem imunodeficiência, foi iniciado tratamento (terapia de indução) com anfotericina B dose de $50 \mathrm{mg}$ intravenoso por quatro semanas. Nesse período a paciente evoluiu com lesão renal aguda, com aumento de escórias nitrogenadas, sendo feita retirada da droga por uma semana, com posterior reintrodução até completar este primeiro esquema terapêutico, sendo feitos LCR de controle, ainda sem negativação de cultura. Concomitante, foi iniciado fluconazol na dose de $400 \mathrm{mg}$ ao dia por 10 semanas. 
A paciente recebeu alta com melhora significativa do estado geral, referindo regressão total dos sintomas. Prescrita terapia de manutenção para domicílio com fluconazol $400 \mathrm{mg}$ a cada 24 horas por seis a 12 meses a depender da avaliação médica ambulatorial em retornos agendados.

O projeto de pesquisa foi submetido à Comissão Interna em Pesquisa (CIP) do hospital responsável pela internação da paciente em questão e posteriormente ao Comitê de Ética em Pesquisa do mesmo hospital. O pesquisador garante o anonimato visto que o projeto está de acordo com os princípios éticos contidos na resolução número 466, de 12 de dezembro de 2012 do Conselho Nacional de Saúde. Os dados serão coletados após aprovação do Comitê de Ética em Pesquisa.

O Termo de Consentimento Livre e Esclarecido (TCLE) do caso foi dispensado visto que for a realizada consulta em banco de dados apenas de prontuário sem qualquer contato com o paciente, mediante as diretrizes e normas reguladoras descritas na Resolução 466/12 do CNS e complementares.

\section{DISCUSSÃO}

Foi apresentado um relato de caso de paciente imunocompetente com sintoma único e predominante de cefaleia há quatro meses com modificação da clínica de migrânea que a mesma possuía previamente, levantando a hipótese de processo meningoencefálico. Com resultado de radiografia de tórax apresentada pela mesma com opacidade mal definida em lobo médio direito, foi levantada a hipótese de infecção fúngica com posterior confirmação de criptococose após realização de punção lombar com coleta e cultura de LCR. Após exames laboratoriais constatou-se que se tratava de uma paciente imunocompetente.

Cryptococcus é uma levedura encapsulada que pode causar doença em pacientes com imunodeficiências, mas também em pacientes imunossuprimidos. A doença criptocócica invasiva é causada por duas cepas patogênicas predominantemente: $C$. neoformans e $C$. gattii. Uma difererença importante entre essas duas espécies além da distribuição geográfica, é que o $C$. gattii não tende a infectar imunossuprimidos de maneira oportunista (GOLDMAN JD, et al, 2010; POLEY M, et al., 2019). O fungo presente em solos, fezes de aves, adentra o organismo humano por via inalatória podendo causar desde uma infecção assintomática até sinais e sintomas de afecção de via aérea inferior, cursando com grave pneumonia podendo, até mesmo, se instalar em outros sítios como sistema nervoso central, pele e olhos (GOLDMAN JD, et al., 2010; ALSPAUGH J A, 2015).

Meningoencefalite por Cryptococcus neoformans é uma causa extremamente rara de doença de sistema nervoso central em pacientes imunocompetentes. Acredita-se que o mecanismo se deve à alta exposição a cepa criptocócica com alta patogenicidade ou à algum déficit imunológico que o paciente possua que não foi detectado. Nesse sentido, álcool, diabetes mellitus, cirrose, doenças autoimunes podem causar esse estado de imunossupressão não detectado, fazendo com que 0 hospedeiro torne-se imunossuprimido temporariamente (POLEY M, et al., 2019)

É bem documentado na literatura que até um terço dos pacientes imunocompetente infectados com Cryptococcus spp. só recebem diagnostico após realização de algum exame de imagem de tórax feitos para outros fins que revelam alguma opacidade mal definida (GOLDMAN JD, et al., 2010). Em relação a doença meningoencefálica em imunocompetente pode se apresentar assintomática em até um terço dos pacientes. No restante, observou-se predominância de cefaleia como sintoma-chave $(73 \%)$, sintomas constitucionais diversos (68\%) e alterações do estado mental (42\%) (POLEY M, et al., 2019).

Ademais, casos de meningococcemia grave com choque séptico fulminante com Cryptococcus são raros e quando presentes, se associam a concomitante infecção pela Neisseria meningitidis (DIENSTMANN G, et al., 2019; PATIL R, et al., 2013). Outra forma de apresentação rara, porem grave, é a associação de hidrocefalia nos casos mais graves e que, inclusive pode ser a primeira manifestação clínica (MAHAJAN K R, et al., 2016).

No caso exposto, a predominância da cefaleia foi bem estabelecida, porém era o único sintoma, dificultando o diagnóstico sindrômico inicial. A paciente apesar de história pregressa que afastava imunodeficiência, apresentou exames laboratoriais normais e negava uso de medicações imunossupressoras. 
A imagem de tórax externa ajudou na possibilidade de solicitação de uma tomografia computadorizada que levantou a suspeita de infecção fúngica com os achados descritos. Após isso, neuroimagem com ressonância magnética confirmou processo inflamatório meningoencefálico. A solicitação do LCR tornou-se mandatória, visto que a hipótese diagnóstica se estabelecia em torno de uma correlação entre afecção pulmonar assintomática com sintoma neurológico, nesse caso uma infecção de etiologia fúngica.

Em relação a análise de líquor cefalorraquidiano nas meningoencefalites, o que é realmente importante para infecção pelo Cryptococcus spp., além dos achados na hora da realização da punção (como pressão de abertura e coloração), é a solicitação de citologia, bioquímica (proteínas, glicose, lactato e ADA), teste de tinta da China, teste de látex para Cryptococcus neoformans e cultura Sabouraud (MACHADO LR, et al., 2013). Ademais, foi visto que não tão somente para diagnóstico, o LCR pode ser utilizado como fator de mau prognóstico quando correlaciona-se nível de consciência prejudicado (analisado pela Escala de Coma de Glasgow) e concentração diminuída de glicose (ZHANG C, et al., 2020).

Outra forma de diagnostico, bem como triagem, vem sendo aplicada em pontos de atendimento em todo o mundo. A utilização do antígeno criptocócico ( $\mathrm{CrAg}$ ) vem sendo utilizado globalmente, sendo identificado por aglutinação em látex com sensibilidade e especificidade de $99 \%$ no sangue e no liquido cefalorraquidiano. Ademais, a triagem do antígeno criptocócico diagnostica em torno de 1 a $15 \%$ de pacientes assintomáticos, sendo proposto tratamento precoce com evidente diminuição da mortalidade (RAJASINGHAM R, et al., 2019).

Após resultado do LCR coletado, pôde-se fechar o diagnóstico etiológico de meningoencefalite por Cryptococcus neoformans na paciente em questão, sendo esta imunocompetente. Após isso, foi iniciado o tratamento com a droga de escolha: anfotericina $B$ na dose de $50 \mathrm{mg}$ ao dia endovenosa, objetivando oito semanas de tratamento com essa droga. Ao final da terapia com anfotericina B, foi introduzido o fluconazol na dose de $400 \mathrm{mg}$ ao dia sendo mantida por pelo menos dez semanas, inclusive após alta hospitalar.

Em relação ao tratamento da neurocriptococose, anfotericina B lipossomal é a droga de escolha, associada a fluconazol ou flucitosina. Essa associação de terapia antifúngica é bem estabelecida na literatura, com melhora da sobrevida nos pacientes que usam a flucitosina (DAY JN, et al., 2014). Todavia, não há disponibilidade no Brasil desta droga, sendo optada pela terapêutica de manutenção com fluconazol na paciente do caso descrito, além de maiores doses de anfotericina $B$ objetivando atingir a dose cumulativa de 2000 a 3000 mg (SCHIAVE et al., 2020). O sucesso do tratamento com o fluconazol deve-se aos níveis séricos e no LCR adequados desta droga. Observou-se que a dose diária ultrapassava a concentração inibitória mínima para o Cryptococcus spp isolados, sendo doses diárias de $400 \mathrm{mg}$ ou mais (máximo $800 \mathrm{mg}$ ) adequadas (SCHIAVE LA, et al., 2018; DAY JN, et al., 2014).

Para finalizar problemática do tratamento, um trabalho publicado no The Lancet em 2013 propôs 10 medidas para melhorar o acesso dos pacientes a terapêutica com antifúngicos, são elas: melhorar estimativa de vida em meningite criptococica, garantir melhor pratica clínica, incluir todos as drogas utilizadas na terapêutica na lista de medicamentos essenciais da Organização Mundial de Saúde, registrar antifúngicos em países sub e não desenvolvidos, aquisição conjunta de antifúngicos, incluir medicações genéricas, propor melhor preço das indústrias farmacêuticas, garantir responsabilidade, garantir licença para desenvolvimento e utilização de novos antifúngicos, otimizar o tratamento já existente, estimular pesquisa a respeito de novos tratamentos (LOYSE A, et al., 2013).

Concluímos que, dada a importância e relevância do tema no diagnostico diferencial das meningoencefalites, o caso descrito nos alertou para a importância de gerar hipóteses diagnosticas mais abrangentes para os diversos sinais e sintomas apresentados pelos pacientes. O estudo foi essencial para compreensão de diagnóstico e tratamento livres de iatrogenias, visto que o bem-estar e resolução da doença apresentada pela paciente eram prioritários. Ademais, o risco de potencial gravidade da doença apresentada, faz necessário diagnósticos mais precisos, de cunho etiológico, visto que tratamentos sindrômicos não resultariam em resolubilidade do quadro.

A redação do relato de caso é uma importante fonte de informações que pode abrir uma serie de possibilidades para diagnósticos diferenciais, fornecendo subsídios para tratamento mais efetivo e correto em algumas situações, como a apresentada (YOSHIDA WB, 2007). 
Após os estudos para realização deste trabalho, foi possível chegar à conclusão de que as meningoencefalites apresentam várias possibilidades etiológicas que não são geradas hipóteses visto a raridade de seu aparecimento na pratica médica. Para a neurocriptococose, o diagnóstico etiológico é importante com anamnese, exame físico e exames complementares bem empregados além de técnicas acessíveis para triagem reduzindo a morbimortalidade dos pacientes. Sendo assim, esse trabalho contribui substancialmente para a área médica, principalmente por ser um caso raro, portanto, pouco estudado por muitos profissionais. Ademais, o maior beneficiado com trabalhos focados em doenças raras é o paciente portador da doença em questão que será tratado da forma correta para evitar desfechos desfavoráveis.

\section{REFERÊNCIAS}

1. ALSPAUGH JA. Virulence mechanisms and Cryptococcus neoformans pathogenesis. Fungal Genetics and Biology, 2015; 78(919): 55-58.

2. DAY JN, et al. Europe PMC Funders Group Combination Antifungal Therapy for Cryptococcal Meningitis. 2014; 368(14):1291-1302.

3. DIENSTMANN G, et al. First case report of fulminant septic shock from meningococcemia associated with Cryptococcus neoformans coinfection in an immunocompetent patient. Medical Mycology Case Reports, 2019; 26(August): 44-46.

4. GOLDMAN JD, et al. Cryptococcosis in the immunocompetent patient. Respiratory Care, 2010; 55(11): 1499-1503.

5. JHA A, et al. Case report: Cryptococcal meningitis in an apparently immunocompetent patient in nepal-challenges in diagnosis and treatment. Wellcome Open Research, 2019; 4: 1-16.

6. LOYSE A, et al. Cryptococcal meningitis: Improving access to essential antifungal medicines in resource-poor countriesThe Lancet Infectious Diseases, 2013.

7. MACHADO LR, et al. O exame do liquido cefalorraquidiano em doenças infecciosas do sistema nervoso: Quando pedir, o que pedir, o que esperar. Arquivos de Neuro-Psiquiatria, 2013; 71(9): 693-698.

8. MAHAJAN KR, et al. Diagnostic Challenges of Cryptococcus neoformans in an Immunocompetent Individual Masquerading as Chronic Hydrocephalus . Case Reports in Neurological Medicine, 2016; 1-7.

9. NIKNAM N, et al. A Case of Recurrent Cryptococcal Meningoencephalitis in an Immunocompetent Female. Case Reports in Infectious Diseases, 2014; 1-3.

10. POLEY M, et al. Cryptococcal Meningitis in an Apparent Immunocompetent Patient. Journal of Investigative Medicine High Impact Case Reports, 2019; 7.

11. RAJASINGHAM R, et al. Cryptococcal Meningitis Diagnostics and Screening in the Era. Journal of Clinical Microbiology, n. September 2018; 1-8.

12. SCHIAVE LA, et al. Fluconazole levels in serum and cerebrospinal fluid according to daily dosage in patients with cryptococcosis and other fungal infectionsBrazilian Journal of Infectious Diseases, 2018.

13. SCHIAVE LA, et al. Minimum concentration of amphotericin B in serum according to the formulation, dose, and daily or prolonged intermittent therapeutic regimen. Revista da Sociedade Brasileira de Medicina Tropical, 2020; 53: 1-7.

14. YOSHIDA WB, Redação do relato de caso. Jornal Vascular Brasileiro, 2007; 6(2): 112-113.

15. ZHANG C, et al. Impaired consciousness and decreased glucose concentration of CSF as prognostic factors in immunocompetent patients with cryptococcal meningitis. BMC Infectious Diseases, 2020; 20(1): 1-6. 